\title{
Robotically assisted mitral valve replacement
}

\author{
Changqing Gao, MD, Ming Yang, MD, Cangsong Xiao, MD, Gang Wang, MD, Yang Wu, MD, \\ Jiali Wang, BS, and Jiachun Li, BS
}

Objective: In the present study, we determined the safety and efficacy of robotic mitral valve replacement using robotic technology.

\begin{abstract}
Methods: From January 2007 through March 2011, more than 400 patients underwent various types of robotic cardiac surgery in our department. Of these, 22 consecutive patients underwent robotically assisted mitral valve replacement. Of the 22 patients with isolated rheumatic mitral valve stenosis ( 9 men and 13 women), the mean age was $44.7 \pm 19.8$ years (range, 32-65). Preoperatively, all patients underwent a complete workup, including coronary angiography and transthoracic echocardiography. Of the 22 patients, 15 had concomitant atrial fibrillation. The surgical approach was through 4 right-side chest ports with femoral perfusion. Aortic occlusion was performed with a Chitwood crossclamp, and antegrade cardioplegia was administered directly by way of the anterior chest. Using 3 port incisions in the right side of the chest and a 2.5- to 3.0-cm working port, all the procedures were completed with the da Vinci S robot.
\end{abstract}

Results: All patients underwent successful robotic surgery. Of the 22 patients, 16 received a mechanical valve and 6 a tissue valve. The mean cardiopulmonary bypass time and aortic crossclamp time was $137.1 \pm 21.9$ minutes (range, 105-168) and $99.3 \pm 17.9$ minutes (range, 80-133), respectively. No operative deaths, stroke, or other complications occurred, and no incisional conversions were required. After surgery, all the patients were followed up echocardiographically.

Conclusions: Robotically assisted mitral valve replacement can be performed safely in patients with isolated mitral valve stenosis, and surgical results are excellent. (J Thorac Cardiovasc Surg 2012;143:S64-7)

Minimally invasive cardiac surgery has emerged as an interesting alternative for a wide variety of surgical procedures. Less-invasive valve surgery has proved even more promising than less-invasive coronary bypass procedures because simpler anastomoses are required. The feasibility and safety of robotic mitral valve replacement (MVR) has been proven in several studies, ${ }^{1-3}$ with the main goals of improved cosmesis and less postoperative discomfort while maintaining the same level of safety and efficacy as with conventional surgery. However, robotically assisted MVR demands different surgical skills in the implant of the prosthesis. Whenever introducing a new technique, it is necessary to determine the incremental risk to the patient compared with the benefit achieved. Few data have been published concerning the outcomes of robotically assisted MVR. ${ }^{4}$

From the Minimally Invasive and Robotic Cardiac Surgery Center, Department of Cardiovascular Surgery, People's Liberation Army General Hospital, Institute of Cardiac Surgery, Beijing, China.

Disclosures: Changqing Gao, Ming Yang, Cangsong Xiao, Gang Wang, Yang Wu, Jiali Wang, and Jiachun Li have nothing to disclose with regard to commercial support.

Presented at The American Association for Thoracic Surgery Mitral Conclave, New York, New York, May 5-6, 2011.

Received for publication April 27, 2011; revisions received Dec 3, 2011; accepted for publication Jan 16, 2012; available ahead of print Feb 3, 2012.

Address for reprints: Changqing Gao, MD, Department of Cardiovascular Surgery, People's Liberation Army General Hospital, Institute of Cardiac Surgery, Beijing,

China, 100853 (E-mail: gachq301@yahoo.com).

0022-5223/\$36.00

Copyright (c) 2012 by The American Association for Thoracic Surgery doi:10.1016/j.jtcvs.2012.01.045
We report the early results for the first 22 consecutive patients who underwent robotically assisted MVR.

\section{METHODS \\ Patients}

From January 2007 through March 2011, more than 400 patients underwent various types of robotic cardiac surgery in our department. Of these patients, 22 consecutive patients underwent robotically assisted MVR. The institutional review board approved the present study, and the 22 patients provided informed consent. Of the 22 patients with isolated rheumatic mitral valve stenosis ( 9 men and 13 women), the mean age was 44.7 \pm 19.8 years (range, 32-65). Preoperatively, all patients underwent a complete workup, including coronary angiography and transthoracic echocardiography. All 22 patients had good left ventricular function. The preoperative characteristics are listed in the Table 1. Two patients were in New York Heart Association class I, 16 in class II, and 4 in class III; 15 patients had concomitant atrial fibrillation (Table 1).

\section{Surgical Technique}

After induction of general anesthesia, all patients were intubated for single-lung ventilation, and a transesophogeal echocardiography (TEE) probe and arterial pressure monitoring line were inserted. Both a central catheter and a $15 \mathrm{~F}$ venous drainage cannula were placed percutaneously into the right internal jugular vein. External defibrillator patches were paced to subtend the maximum cardiac mass. Each patient was positioned with the right side of the chest elevated approximately $30^{\circ}$ and with right arm tucked at the side. After systemic heparinization, femoral arterial $(18 \mathrm{~F}$ or $20 \mathrm{~F})$ and venous $(21 \mathrm{~F}$ or $23 \mathrm{~F})$ cannulation (Metronic, Minneapolis, Minn) were performed through a 2-cm transverse right groin incision cutdown with the Seldinger guidewire method and TEE guidance. Bicaval venous drainage was instituted through the jugular and femoral/inferior vena cava cannulas. 


\section{Abbreviations and Acronyms \\ ICS = intercostal space \\ MVR $=$ mitral valve replacement \\ TEE $=$ transesophogeal echocardiography}

The camera cannula was placed in the right side, 2 to $3 \mathrm{~cm}$ lateral to the nipple in the fourth intercostal space (ICS). A $2.5-$ to $3.0-\mathrm{cm}$ incision was used as a working port in the same ICS for the patient-side surgeon. The soft tissue retractor was inserted into this port, and no other rib retractor was used (Figure 1). The da Vinci instrument arms were inserted through three $0.8-\mathrm{cm}$ trocar incisions in the right side of the chest. The right instrument arm was generally poisoned lateral to the working port in the sixth ICS. The left instrument arm was positioned medially and cephalad to the working port in the second ICS. The fourth trocar arm was placed in the midclavicular line in the fifth ICS. All resection and suturing of the mitral valve and atrial closure were completed with the da Vinci S robot.

Cardiopulmonary bypass was initiated with kinetically assisted bicaval venous drainage. The aorta was occluded with a Chitwood crossclamp (Scanlan International, Minneapolis, Minn) using the midaxillary line in the fourth ICS. Antegrade cold blood cardioplegic solution was administered directly through the anterior chest (second ICS) with a 14F angiocatheter, by which de-airing was conducted, and repeat doses could be given when necessary. TEE was used routinely to monitor the position of the angiocatheter and assist with de-airing. Carbon dioxide was insufflated continuously into the operative field for air displacement. The left atrium was then opened parallel to the interatrial septum. All MVRs were performed using standard techniques. The diseased mitral valve was completely excised, and the posterior leaflets were preserved whenever possible. Appropriate sizing was performed, and everting, double-armed, mattress sutures with Teflon pledgets were placed counterclockwise from the 11-o'clock position. Every stitch was fixed sequentially outside with a small hemostat. Usually 10 to 12 sutures were needed (Figure 1). Once the sutures were placed in the prosthesis sewing ring outside the chest, the prosthesis was lowered into the chest and positioned, and the knots were tied using the knot pusher through the incision (Figure 2). The atriotomy was closed with 4-0 polytetrafluoroethylene running suture (W. L. Gore \& Associates, Flagstaff, Ariz). Before the Chitwood crossclamp was released, meticulous intracardiac de-airing was conducted through the angiocatheter of cardioplegia, and then the aorta was unclamped. The patient was weaned from cardiopulmonary bypass, and the cardioplegia site was closed with an

TABLE 1. Patient characteristics $(n=22)$

\begin{tabular}{lc}
\hline \multicolumn{1}{c}{ Characteristic } & Value \\
\hline Age (yr) & \\
Mean & $44.7 \pm 9.8$ \\
Range & $32-65$ \\
Gender (n) & \\
Male & $9(35)$ \\
Female & $13(65)$ \\
Mean ejection fraction (\%) & $50.2 \pm 13.8$ \\
New York Heart Association class & \\
I & $2(10)$ \\
II & $14(70)$ \\
III & $4(20)$ \\
Sinus rhythm (n) & $5(25)$ \\
Atrial fibrillation (n) & $15(28)$ \\
\hline
\end{tabular}

Data in parentheses are percentages.

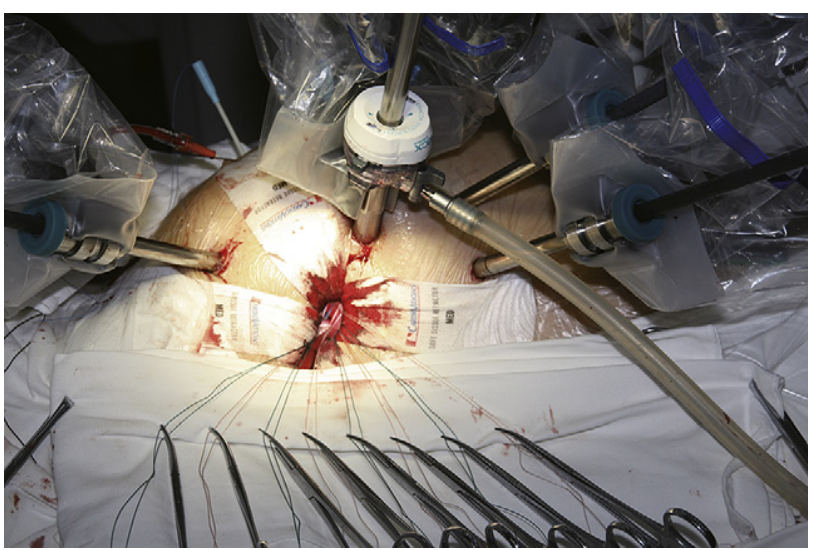

FIGURE 1. da Vinci setup for mitral valve replacement. Every stitch was fixed sequentially outside with a small hemostat.

extracorporeal knot tied through the working port, and chest tubes were inserted. The prosthesis function was confirmed by TEE.

The data are shown as the mean \pm standard error of the mean (Figures 1 and 2).

\section{RESULTS}

All patients underwent successful robotically assisted MVR. Of the 22 patients, 16 received a mechanical valve (St Jude Medical, St Paul, Minn) and 6 received a bioprosthetic valve (Cosgrove-Edwards; Edwards Lifesciences, Irvine, Calif), and all patients recovered uneventfully. The mean cardiopulmonary bypass time and aortic crossclamp time was $137.1 \pm 21.9$ minutes (range, 105-168) and 99.3 \pm 17.9 minutes (range, 80-133), respectively. As shown in Figure 2, both the crossclamp and bypass times decreased significantly with experience. Intraoperative TEE revealed appropriate prosthesis function in all patients. One patient had effusion in the right hemithorax. The mean intensive

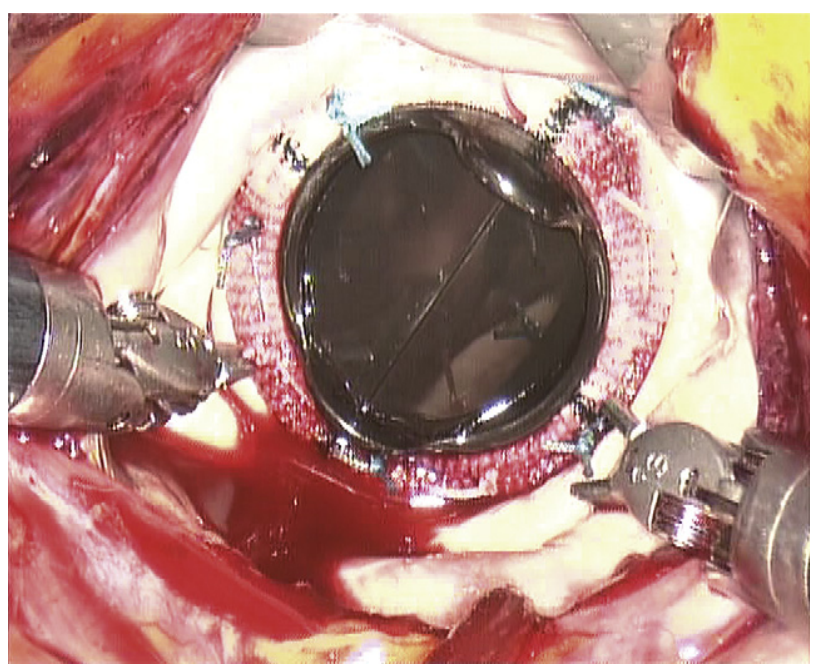

FIGURE 2. The mechanical valve is set into the right position and tied through the working port. 
care unit stay was 1.5 days (range, $1-3$ ). In the entire series, no device-related complications, stroke, peripheral ischemic event, or operative deaths occurred, and no incisional conversions were required. After surgery, all patients were followed up echocardiographically, and no patient had a paravalvular leak. The mean follow-up time was $12.1 \pm 6.6$ months (Figure 3).

\section{DISCUSSION}

The most common surgical approach to the mitral valve requires the surgeon to saw open the breastbone and spread the edges apart to gain direct access to the heart. Although this approach provides excellent access to the heart, the resulting wound requires several months to heal completely, resulting in an extended recovery period with substantial activity restrictions, and can be subject to serious complications, including infection, breakdown, and even death. In contrast, robotic cardiac surgery can extend the ability of the surgeons, performing MVR using a 3-cm working port without rib resection and retraction, toward new challenges, albeit with the potential danger of performing suboptimal surgery because of the learning curve issue.

After more than 400 cases of various types of robotic cardiac surgery, ${ }^{5-9}$ we believe that the da Vinci Surgical system can be considered the best surgical solution to the philosophic approach of minimally invasive cardiac surgery. The 3-dimensional digital vision system enables natural depth perception with high-power magnification $(10 \times)$. A perfect view was obtained of all cardiac chambers, the mitral valve, and the subvalvular apparatus, especially in patients with a small left atrium.

Although many reports have described the excellent results of robotically assisted mitral valve repair using the da

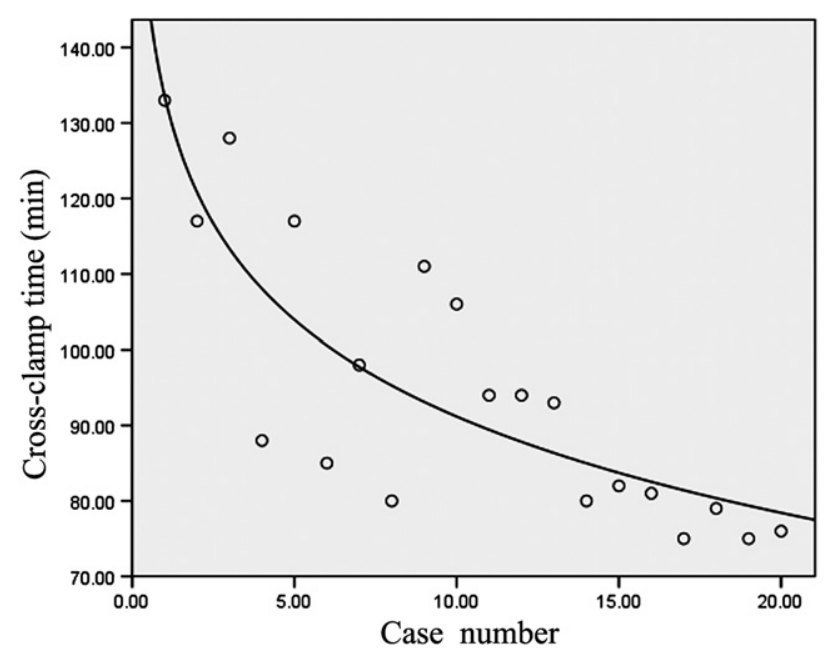

FIGURE 3. The learning curve for the crossclamp time of robotic mitral valve replacement (learning curve for crossclamp time: $\mathrm{y}(\mathrm{min})=132.757$ $\left.-17.82 \ln (\mathrm{x}) ; r^{2}=0.718 ; P<.01\right)$.
Vinci Surgical system, ${ }^{1-3}$ few data are available on robotically assisted MVR. ${ }^{4}$ All our patients had satisfactory clinical outcomes, as demonstrated by the complete echocardiographic follow-up. No late thromboembolic complications or paravalvular leaks developed. Intraoperative contamination must be considered when performing extrathoracic knots and introducing the prosthetic ring through the narrow working port, although no cases of either native or prosthetic mitral valve endocarditis were identified during follow-up in our patients.

The suture techniques for robotic MVR are very different from those in the open technique. For all mechanical and bioprosthetic valves, we used everting, double-armed mattress sutures with Teflon pledgets counterclockwise from the 11-o'clock position to prevent any possibility of subannular obstruction of the valve leaflets by protruding tissue and to prevent 1 suture from crossing with another. Every stitch was fixed sequentially outside with a small hemostat. Usually 10 to 12 sutures were needed (Figure 1). Once the sutures were placed sequentially in the prosthesis sewing ring outside the chest, the prosthesis was lowered into the chest and positioned, and the knots were tied with the knot pusher through the incision. When the knots are tied with the knot pusher, the console surgeon must confirm the knot tightness 1 by 1 , and in the case of a knot not being tight, the knot tightness requires robotic assistance. Recently, we started using the Cor-Knot (LSI Solution, Victor, NY) to tie the knots during robotic MVR. This has resulted in a shorter operative time, and the preliminary results have been satisfactory.

It is believed that any new surgical approach requires a learning curve, and robotic cardiac surgery is no exception. We believe that robotic cardiac surgery requires real teamwork and surgeons must overcome a substantial learning curve. Our leaning curve is showed graphically in Figure 3. The mean crossclamp time was significantly shortened with surgical experience as denoted by the number of operations. Our learning curve was truncated because the console surgeon simultaneously performed a large volume of various types of other robotic cardiac surgeries with the same team within a relative short period. Furthermore, since we started our robotic surgical program, our robotic team has not changed. Our experience has demonstrated that with a well-trained robotic team and after a substantial learning curve, optimal results can be achieved with robotic surgery. $^{5-9}$

In conclusion, the present results suggest that robotically assisted MVR for patients with isolated mitral valve stenosis is safe. The 3-dimensional digital vision system provides a perfect view of the mitral valve and the subvalvular apparatus in patients in whom limited exposure prohibits direct vision. This method also provides the unique opportunity to observe experienced surgeons at work, shortening the learning curve and avoiding mistakes. 


\section{References}

1. Atluri P, Woo YJ. Minimally invasive robotic mitral valve surgery. Expert Rev Med Devices. 2011;8:115-20.

2. Lehr EJ, Rodriguez E, Chitwood WR. Robotic cardiac surgery. Curr Opin Anaesthesiol. 2011;24:77-85

3. Mihaljevic T, Jarrett CM, Gillinov AM, Williams SJ, DeVilliers PA, Stewart WJ, et al. Robotic repair of posterior mitral valve prolapse versus conventional approaches: potential realized. J Thorac Cardiovasc Surg. 2011;141:72-80. e1-4.

4. Murphy DA, Miller JS, Langford DA, Snyder AB. Endoscopic robotic mitral valve surgery. J Thorac Cardiovasc Surg. 2006;132:776-81.
5. Gao C, Yang M, Wu Y, Wang G, Xiao CS, Liu HB, Lu CY. Hybrid coronary revascularization by endoscopic robotic coronary artery bypass grafting on beating heart and stent placement. Ann Thorac Surg. 2009;87:737-41.

6. Gao C, Yang M, Wu Y, Wang G, Xiao CS, Zhao Y, et al. Early and midterm results of totally endoscopic coronary artery bypass grafting on the beating heart. J Thorac Cardiovasc Surg. 2011;26:843-9.

7. Gao C, Yang M, Wang G, Wang JL, Xiao CS, Wu Y, et al. Totally endoscopic robotic atrial septal defect repair on the beating heart. Heart Surg Forum. 2010;13:140-3.

8. Gao C, Yang M, Wang G. Totally endoscopic robotic ventricular septal defect repair. Innovations. 2010;5:278-80

9. Gao C, Yang M, Wang G, Xiao CS, Wu Y, Li JC. Excision of atrial myxoma using robotic technology. J Thorac Cardiovasc Surg. 2010;139:1282-5. 